\title{
Massimo Mori
}

\section{Friede und Föderalismus bei Kant ${ }^{1}$}

\section{1. , Westfälisches Modelk und , domestic analogy}

Unmittelbar nach dem Ersten Weltkrieg nahm der ehrgeizige Versuch Gestalt an, durch ein Bündnis der Völker, das Streitigkeiten mit Hilfe eines internationalen Schiedsspruchs schlichten sollte, den künftigen Frieden zu fördern. Dem Völkerbund, der aus diesem Projekt hervorging, war indes kein langes Leben beschieden und vor allem erwies er sich als untaugliches Werkzeug für die Verwirklichung des gesetzten Ziels. Ein gelungenerer Versuch war die Gründung der Vereinten Nationen (UNO) nach dem Zweiten Weltkrieg, die nach wie vor tätig ist, auch wenn vor allem in den letzten Jahren ihre institutionelle Schwäche und ihre geringe Durchsetzungskraft in Krisensituationen immer deutlicher zu Tage getreten sind. Es scheint, als habe sich Woodrow Wilson, der noch vor Kriegsende für die Gründung des Völkerbunds eintrat, an Kant inspiriert, obwohl er in seinen Schriften nur sehr selten und in allgemeiner Form auf den großen Philosophen Bezug nimmt. Noch indirekter ist die kantische Urheberschaft der UNO, die aus einer umfänglichen diplomatischen Aktion entstand, in der sich nur schwer philosophische Einflüsse ermitteln lassen. Auf konzeptioneller Ebene bildet Kants Projekt Zum ewigen Frieden aber unzweifelhaft das Vorbild für beide Institutionen, weil es mit ihnen die ebenso ehrgeizige wie schwer zu verwirklichende Idee teilt, dass ein institutionalisiertes, also von einem zentralen Organismus abhängiges Rechtsverhältnis zwischen allen Völkern der Erde herstellbar sei.

In Wirklichkeit war Kants Bezugsmodell ein Philosoph, dem nichts ferner stehen konnte als der Gedanke eines ewigen Friedens unter den Völkern, nämlich Thomas Hobbes. In zwei Punkten stimmen der Autor von De Cive und Leviathan und Kant überein. Erstens konzipieren beide den Naturzustand als Kriegszustand, in dem es nicht zwangsläufig zu Konflikten und realen Zusammenstößen kommt, der Krieg aber potenziell immer möglich ist, weil es an einer unparteilichen Autorität fehlt,

1 Der vorliegende Text spiegelt einen Vortrag wieder, der bei der Siemens-Stiftung München am 14. 06. 2006 gehalten wurde. Es geht um ein work in progress, der im Rahmen eines umfangreicheren, von der Alexander von Humboldt-Stiftung und der Universität Turin befördeten Forschungsprojekts über Kants internationales Denken steht. Für die Diskussion der einschlägigen Sekundärliteratur wird auf die endgültige Fassung hingewiesen - Alle Kant-Zitate stammen aus der Akademie-Ausgabe: Kantıs Gesammelte Schriften, Berlin-Leipzig, 1910 ff. Im Text werden Band, Seite und Zeile im Klammern angegeben. 
die Streitigkeiten mit rechtlichen Mitteln schlichten würde 2. Die zweite Analogie besteht in der gemeinsamen Überzeugung beider Autoren, dass man aus dem natürlichen Kriegszustand herausfinden muss (exeundum e statu naturali), indem man vom Zustand der natürlichen Freiheit zu einem Zustand von gesetzlichem Zwang übergeht. Für Hobbes wie für Kant konnte dies einzig und allein durch die Schaffung der bürgerlichen Gesellschaft gelingen, auch wenn die Bedingungen des Gesellschaftsvertrags, auf dem sie basiert, und folglich die Natur des daraus entstehenden Staates für beide völlig andere waren.

Damit sind die Analogien aber auch schon zu Ende und es tritt stattdessen ein sehr erheblicher Unterschied hervor. Hobbes zieht die Ausweitung des Gesellschaftsvertrags von einer einzelstaatlichen zur zwischenstaatlichen Ebene nicht in Betracht. Mit anderen Worten ist der Staat für Hobbes die höchste Form von politischer Institution, über die hinaus es keine andere Obrigkeit gibt. Der Souverän ist mit jener schon von Jean Bodin theoretisierten potestas absoluta ausgestattet, die ihn allein Gott unterstellt. Das von Hobbes erarbeitete Denkmodell entspricht übrigens der internationalen Realität seiner Zeit. Nach dem Westfälischen Frieden von 1648, mit dem de facto die übernationalen politischen Funktionen von Papsttum und Kaiserreich schwanden, bildete sich das jus publicum europaeum heraus, mit dem die internationale Ordnung nun auf ein System souveräner Staaten gegründet wurde, also auf das Gleichgewicht zwischen Nationen, die sich als gleichberechtigt betrachteten und wechselseitig ihre Unabhängigkeit anerkannten.

Zwar waren die meisten Vertreter der Naturrechtsschule im Unterschied zu Hobbes, mit dem sie doch die These vom vertraglichen Ursprung des Staates teilten, der Ansicht, dass es ein nicht nur die Individuen, sondern auch die Staaten betreffendes Naturrecht gebe. Das internationale Naturrecht würde die Staaten an Gewaltanwendung, also an Kriegen, hindern, es sei denn zur Selbstverteidigung oder Zurückweisung eines Angriffs (vim per vim repellere licet, wie der alte Spruch von Ulpian lautet). Die Gewaltanwendung wäre nur insoweit gerechtfertigt als sie der Wiederherstellung der verletzten Rechtsordnung dient. Auf diesem Gedanken beruht seit Thomas von Aquin die Lehre vom »gerechten Krieg«. Aber gerade weil das internationale Naturrecht den Naturrechtsphilosophen ganz und gar ausreichend erschien, um die zwischenstaatlichen Beziehungen zu regeln, hielten sie es mit dem Prinzip der absoluten Souveränität für durchaus vereinbar. Die souveränen Staaten selbst hatten es anzuwenden. Deshalb schlossen die Naturrechtsphilosophen - genau wie Hobbes - die Notwendigkeit einer Ausdehnung des Gesellschaftsvertrags von der individuellen auf die staatliche Ebene kategorisch aus. Die einzige Ausnahme bildete Christian Wolff, der eine die besonderen civitates umschließenden civitas maxima voraussetzte ${ }^{3}$. Allerdings bestätigt die Ausnahme die

2 Vgl. Thomas Hobbes, De cive, I, 12; Leviathan, I, XIII; Immanuel Kant, Zum ewigen Frieden, VIII, 348, 4-5 / 349, 1-2.

3 Christian Wolff, Institutiones juris naturae et gentium, Halle-Magdeburg, 1750, Prolegomena, \S 9-21. Die civitas maxima ist aber von Wolff als reine Fiktion der Vernunft verstanden. 
Regel: Sein Schüler Emer de Vattel, Verfasser eines viel gelesenen Droit de gens, das als Handbuch in der europäischen Diplomatie weite Verbreitung fand, ging zu der Lehre von der civitas maxima entschieden auf Abstand, obgleich er dem Lehrer in allen wichtigen Fragen bis ins Kleinste folgte. In seinen Augen war sie von Grund auf falsch, weil sie den Staaten ihre Unabhängigkeit nahm ${ }^{4}$. In der naturrechtsphilosophischen Perspektive, wie sie die politische Kultur des 17. und 18. Jahrhunderts allgemein beherrschte, war die Achtung des internationalen Rechts somit in das Ermessen der einzelnen souveränen Staaten gestellt, ohne Anrufungsmöglichkeit irgendeiner höheren Rechtsinstanz. Das vor Kant dominierende politische Modell, auf das die Politologen noch heute unter dem Begriff » Westfälisches Modell« Bezug nehmen, fußt demnach wesentlich auf dem Prinzip internationaler Anarchie.

Diesem Hobbessschen und allgemein naturrechtsphilosophischen Modell setzte Kant die von den heutigen Politologen als domestic analogy bezeichnete Theorie entgegen. Dieser zufolge ist die Situation zwischen den Staaten im Naturzustand die gleiche wie zwischen den Individuen: Es handelt sich jeweils um einen Kriegszustand. Daher muss das Vertragsmodell von der individuellen auf die staatliche Ebene ausgedehnt werden.

»Völker als Staaten können wie einzelne Menschen beurtheilt werden, die sich in ibrem Naturzustande (d. i. in der Unabhängigkeit von äußern Gesetzen) schon durch ibr Nebeneinandersein lädiren, und deren jeder um seiner Sicherheit willen von dem andern fordern kann und soll, mit ibm in eine der bürgerlichen ähnliche Verfassung zu treten, wo jedem sein Recht gesichert werden kann "(VIII, 354, 3-8).

Gehen wir also davon aus, dass die Staaten ebenso wie die Individuen in eine Art internationale Gesellschaft treten müssen, um aus dem natürlichen Kriegszustand herauszufinden, so stellt sich folgende Frage: Beinhaltet die Ausweitung des Vertragsmodells von der zwischenmenschlichen zur zwischenstaatlichen Ebene eine vollkommene oder eine unvollkommene Analogie zwischen beiden Ebenen? Die Alternative zieht höchst unterschiedliche Folgen nach sich. Ist die Analogie vollkommen, so müssen die Staaten einer einzigen Zentralgewalt unterstehen, wie die Bürger sich einer einzigen Regierung unterwerfen. Mit Kant gesprochen wäre die daraus hervorgehende Staatengesellschaft also ein »Völkerstaat «. Wenn die Analogie hingegen nur unvollkommen ist, wenn das auf die Individuen angewandte Vertragsmodell also nur bis zu einem gewissen Punkt auf die Staaten ausgedehnt werden kann, dann gehen diese einfach in einem Organismus internationaler Koordination zusammen, der über keinerlei Zwangsgewalt (also über keine Zentralregierung) verfügt, sondern die nationale Unabhängigkeit und Souveränität der einzelnen Mitgliedsstaaten unangetastet lässt. In diesem Fall gelangt man nicht zu einem Völkerstaat, sondern zu einem bloßen »Völkerbund «. Konzeptionell unterscheiden sich die beiden Lösungen durch das Vorhandensein bzw. Nichtvorhandensein einer

4 Emer de Vattel, Le droit des gens, London, 1758, Préface, I, pp. XVII-XVIII.

ZfP 53. Jg. 4/2006 
Zentralregierung, doch können sie einander im Hinblick auf ihren Zwangscharakter durchaus sehr annähern. Die Gewalten des Völkerstaats können sich in manchen Fällen auf wenige für die Friedenssicherung notwendige außenpolitische Kompetenzen beschränken ${ }^{5}$, während die Befugnisse des Völkerbunds von der bloßen politischen Empfehlung bis zu einem mit vereinten militärischen Kräften ausgeübten Zwang reichen können. Jedenfalls wird der internationale Friede besser durch den Völkerstaat garantiert, Freiheit und Unabhängigkeit der einzelnen Staaten durch den Völkerbund.

\section{Zwischen Völkerbund und Völkerstaat}

Kants Lösung des angesprochenen Problems durchläuft eine parabelartige Entwicklung. Obwohl er grundsätzlich stets dem Völkerbund den Vorzug gab, gewann bis Anfang der 1790er Jahre die These des Völkerstaates an Gewicht; danach sprach er sich hingegen immer nachdrücklicher zugunsten des Völkerbunds aus.

In der Idee zu einer allgemeinen Geschichte in weltbürgerlicher Absicht (1784) steht die genannte Alternative noch nicht zur Debatte. Die einzige vorgeschlagene Lösung ist die des Völkerbunds. Zwar macht Kant hier keine konkreten Angaben zur institutionellen Struktur der Föderation, doch ist sie seinen Äußerungen zufolge ermächtigt, »nach Gesetzen des vereinigten Willens « zu beschließen und diesen Willen mit Hilfe der »vereinigten Macht« (V, 24, 26-27) durchzusetzen. Sein Hinweis auf den foedus anphictyonum scheint auf den Bündnispakt der griechischen poleis anzuspielen. Die Zwangsgewalt der Föderation ist mithin nicht durch ihre institutionellen Organe gegeben, sondern durch die tatsächliche Fähigkeit zur Umsetzung gemeinsam getroffener Entscheidungen unter Vereinigung der einzelnen Kräfte. Folglich denkt Kant lediglich an einen Völkerbund - auch in späteren Werken die einzige von ihm akzeptierte Lösung -, schreibt diesem Bund jedoch de facto weit reichende Zwangsgewalt zu, die ihm eine ähnliche Durchsetzungskraft wie einem Völkerstaat verleiht.

In der Schrift Über den Gemeinspruch: Das mag in der Theorie richtig sein, tangt aber nicht für die Praxis (1793) unterscheidet Kant erstmals eindeutig - wenigstens was die gedruckten Werke anbelangt - zwischen einer »weltbürgerlichen Verfassung « als »weltbürgerliches gemeines Wesen unter einem Oberhaupt«, das heißt einem Völkerstaat, und »ein[em] rechtliche[n] Zustand der Föderation nach einem gemeinschaftlich verabredeten Völkerrecht«, das heißt einem Völkerbund (VIII, 311, 4-5). Die Entscheidung für eine der beiden Möglichkeiten ist durch den Konflikt zwischen dem, was die »Theorie« vorschreibt und dem, was die »Praxis« nahelegt, bedingt. Die auf das »Völkerrecht« bezogene Theorie ist unmissverständlich: Um den Kriegszustand zu überwinden, ist »kein anderes Mittel, als auf öffentliche

5 Vgl. Z. B. die heute von Otfried Höffe vorgeschlagene Teorie des »extrem minimalen Weltstaates « (Otfried Höffe, »Völkerbund oder Völkerrepublik « in: Immanuel Kant, Zum ewigen Frieden, hrsg. v. Otfried Höffe, Berlin 1995, S. 109-32). 
mit Macht begleitete Gesetze, denen sich jeder Staat unterwerfen müßte, gegründetes Völkerrecht (nach der Analogie eines bürgerlichen oder Staatsrechts einzelner Menschen) möglich « (VIII, 312, 25-29). Die Lösung wäre demnach der Völkerstaat. Aber in der »Praxis « wenden diejenigen, die sich gegen die Möglichkeit einer Anpassung der Praxis an die Theorie wehren, ein, dass die Staaten sich niemals solchen Zwangsgesetzen beugen werden; sie leugnen also die Möglichkeit eines Völkerstaats. Dagegen spricht Kant sich gemäß der allgemeinen These der Schrift, wonach Erwägungen praktischen Nutzens die Gültigkeit der theoretischen Sätze nicht beeinflussen dürfen, zugunsten des Völkerstaats aus:

"Ich meinerseits vertrane dagegen doch auf die Theorie, die von dem Rechtsprinzip ausgebt, wie das Verbältniß unter Menschen und Staaten sein soll, und die den Erdengöttern die Maxime anpreiset, in ibren Streitigkeiten jederzeit so zu verfahren, dass ein solcher allgemeiner Völkerstaat dadurch eingeleitet werde, und ibn also als möglich (in praxi), und daß er sein kann, anzunehmen" (VIII, $313,7-12)$.

Im Jahr 1795 verfasste Kant das Werk, das die eingehendste Auseinandersetzung mit dem Problem von Krieg und Frieden darstellt: Zum ewigen Frieden. Das Problem wird darin ähnlich gestellt wie in der Schrift von 1793, aber Kants Position erscheint genau umgekehrt. Nach wie vor steht der Völkerstaat auf der Seite der Theorie und der Völkerbund auf der Seite der Praxis, doch pflichtet Kant jetzt den Erwägungen praktischen Nutzens bei, womit er klar hinter die These von 1793 zurückgeht. Er unterscheidet zwischen einer Position »in thesi«, also aufgrund des von der Vernunft Gesetzten (titemi), und einer Position »in ypothesi«, die von nicht auf die Vernunft zurückführbaren empirischen Voraussetzungen ausgeht. »In thesi« kann an der vollkommenen Analogie zwischen Staaten und Individuen und somit an der Notwendigkeit eines »Völkerstaates« kein Zweifel bestehen:

"Für Staaten im Verbältnisse unter einander kann es nach der Vernunft keine andere Art geben, aus dem gesetzlosen Zustande, der lauter Krieg enthält, herauszukommen, als daß sie eben so wie einzelne Menschen ibre wilde (gesetzlose) Freibeit aufgeben, sich zu öffentlichen Zwangsgesetzen bequemen und so einen (freilich immer wachsenden),Völkerstaat, (,Civitas gentium<), der zuletzt alle Völker der Erde befassen würde, bilden" (VIII, 357, 5-11).

Was Kant aber "in thesi«, also vom theoretischen Standpunkt, weiterhin richtig erscheint, stellt sich ihm in der Praxis als nicht umsetzbar dar, weil die Staaten nämlich von der »Ypothesis « ausgingen, nicht auf ihre Unabhängigkeit »nach ibrer Idee des Völkerrechts « (meine Hervorhebung) verzichten zu müssen. Auch Kant akzeptiert diese »Voraussetzung « und meint, man müsse sich - (»wenn nicht alles verloren werden soll«) - »an d[er] Stelle der positiven Idee einer Weltrepublik « mit dem "negative[n] Surrogat eines den Krieg abwehrenden, bestehenden und sich immer ausbreitenden Bundes (VIII, 357, 13-16) zufrieden geben, auch wenn die besagte Idee einer Weltrepublik in thesi rational richtig wäre. Schon der Überschrift nach setzt sich der zweite Definitvartikel zum ewigen Frieden daher nur »einen Födera- 
lism freier Staaten « (meine Hervorhebung) zum Ziel. Das internationale Recht zielt »lediglich auf Erhaltung und Sicherung der Freiheit eines Staats für sich selbst und zugleich anderer verbündeten Staaten, ohne daß diese doch sich deshalb (wie Menschen im Naturzustande) öffentlichen Gesetzen und einem Zwange unter denselben unterwerfen dürfen« (VIII, 356, 11-14).

Die größte Abneigung gegen den Völkerstaat samt der daraus folgenden institutionellen Schwächung des internationalen Organismus für die Erhaltung des Friedens zeigt Kant in der Metaphysik der Sitten von 1798 (Rechtslebre, $\$ 61$ ). Tatsächlich hält er auch in diesem Werk an dem Prinzip fest, wonach der Völkerstaat theoretisch betrachtet die einzig wahre Lösung der Friedensfrage darstellen würde:

»Da der Naturzustand der Völker eben so wobl als einzelner Menschen ein Zustand ist, aus dem man herausgehen soll, um in einen gesetzlichen zu treten: so ist vor diesem Ereignis alles Recht der Völker und alles durch den Krieg erwerbliche oder erbaltbare äussere Mein und Dein der Staaten blo $\beta$ sprovisorisch und kann nur in einem allgemeinen Staaten-Verein (analogisch mit dem, wodurch ein Volk Staat wird) peremptorisch gelten und ein wahrer Friedenszustand werden " (VI, 350, 6-12).

Doch trotz dieser klaren Konzession an die Theorie vertritt Kant die Ansicht, dass der internationale Organismus für die Erhaltung des Friedens »keine souveräne Gewalt (wie in einer bürgerlichen Verfassung), sondern nur eine Genossenschaft (Föderalität) enthalten müsse« (VI, 344, 18-19). Der Verein, der keinerlei Einmischung in die Innenpolitik seiner Mitglieder zulassen darf, soll temporärer Art sein und muss folglich von Zeit zu Zeit erneuert werden. Kant so äußert über die internationale Institution, die er mit der 1719 in Den Haag einberufenen Versammlung der Generalstaaten vergleicht: »Man kann einen solchen Verein einiger Staaten, um den Frieden zu erhalten, den permanenten Staatenkongre $\beta$ nennen, zu welchem sich zu gesellen jedem benachbarten unbenommen bleibt (VI, 350, 23-25). Um jeglichen Zweifel auszuräumen, setzt er erläuternd hinzu, dass unter Kongress »nicht eine solche Verbindung, welche (wie die der amerikanischen Staaten) auf einer Staatsverfassung gegründet und daher unauflöslich ist«, sondern einfach »eine willkürliche, zu aller Zeit auflösliche Zusammentretung verschiedener Staaten« (VI, 351, 1-4) zu verstehen sei.

\section{Das»Postulat des öffentlichen Rechts"}

Bevor wir untersuchen, aus welchen Gründen Kants Skepsis gegenüber dem Völkerstaat von 1793 an immer größer wurde, sollten wir uns fragen, ob diese Position mit dem allgemeinen Rahmen der kritischen Philosophie und namentlich mit der transzendentalen Rechtsauffassung vereinbar ist. Das Recht ist für Kant »der Inbegriff der Bedingungen, unter denen die Willkür eines jeden mit jedermanns Freiheit nach einem allgemeinen Gesetze zusammen bestehen kann« (VI, 230, 24-26). Mit anderen Worten ist das Recht das von der reinen Vernunft (in ihrem praktisch- 
rechtlichen Gebrauch) festgesetzte allgemeine System der Freiheiten, aufgrund dessen jedermanns äußere Freiheit mit der aller anderen vereinbar wird. Obwohl das von der reinen Vernunft bestimmte Rechtssystem absoluten normativen Wert hat, besitzt es indes keinerlei tatsächliche Macht, solange es nicht in ein positives Rechtssystem übersetzt wird, also mit Zwangsgewalt ausgestattetes Gesetz wird: »Recht und Befugnis zu zwingen bedeuten also einerlei«(VI, 232, 29). Mit Kant gesprochen hat das »Privatrecht « (natürliches Recht) nur »provisorischen « Wert; allein das »öffentliche Recht« (positives Recht) ist »peremptorisch«.

Deshalb beruht das »öffentliche Recht« nach Kant auf einem »Postulat«:

»Du sollst im Verbältnisse eines unvermeidlichen Nebeneinanderseins mit allen anderen aus jenem heraus in einen rechtlichen Zustand, $d$. $i$. den einer austheilenden Gerechtigkeit, übergehen "(VI, 307, 9-11).

Anders gesagt: Wenn die Menschen die Möglichkeit haben, in Wechselbeziehungen zu treten und sich folglich auch gegenseitig zu schaden, so müssen sie notwendigerweise aus dem Naturzustand heraustreten, der ein Kriegszustand ist, um stattdessen in einen rechtlichen Zustand zu treten, der ein Zustand des Friedens ist. Nun haben die Menschen, so Kant, stets die Möglichkeit, miteinander in Beziehung zu treten, weil die Erde rund, ihre Oberfläche also nicht unendlich ist; außerdem können nunmehr auch große Distanzen leicht zurückgelegt werden - das 18. Jahrhundert ist ein Jahrhundert der großen Entdeckungsreisen -, es gibt also keine inneren Schranken mehr, die die Völker voneinander trennen. Daraus ergibt sich das im Naturzustand allen kraft Vernunft verliehene Recht, die anderen zum Hinaustreten aus diesem Zustand zu zwingen, um sich gemeinsam eine »rechtliche Verfassung « zu geben, in der das Recht nicht nur "provisorisch «, als im Naturrecht enthaltene Vernunftvorgabe, sondern »peremptorisch « durch den mit dem positiven Recht gesetzten $Z_{\text {wang gilt. }}$

Das öffentliche Recht gliedert sich seinerseits in drei Ebenen. Es betrifft 1. die Beziehungen zwischen Individuen innerhalb eines Staates im Sinne der bürgerlichen Verfassung (Staatsrecht, ius civitatis); 2. die Beziehungen zwischen Staaten (Völkerrecht, ius gentium); 3. die Beziehungen zwischen Staaten und Einzelmenschen (weltbürgerliches Recht, ius cosmopoliticum) (VIII, 349, 25-35). Folglich müsste das »Postulat des öffentlichen Rechts « auf allen drei genannten Ebenen Gültigkeit haben, das heißt, zwischen den verschiedenen Ebenen müsste eine perfekte Analogie herrschen. Auch wenn die miteinander in Beziehung tretenden Subjekte von Fall zu Fall andere sind - Individuen mit Individuen, Staaten mit Staaten, Individuen mit Staaten - müsste stets die Regel gelten, derzufolge zwei Subjekte, die in Wechselbeziehungen kommen, aus dem Naturzustand herausfinden und in einen gesetzlichen Zustand übergehen müssen. Aber genau in diesem Punkt gerät Kant gewisserweise in Widerspruch zu sich selbst, weil er die Befolgung des »Postulats « de facto nur für die erste Ebene fordert, auf der die einzelnen Bürger der Zwangsgewalt des Staates unterstehen. Auf der zweiten Ebene, der der internationalen Beziehungen, würde die genaue Anwendung des »Postulats « nämlich unweigerlich zur Befürwortung des »Völkerstaats « führen, zu der Kant - wie wir gesehen haben - nicht bereit ist. 
Die dritte Ebene endlich, die des weltbürgerlichen Rechts, betrifft ausschließlich das »Besuchrecht, welches allen Menschen zusteht, sich zur Gesellschaft anzubieten« (VIII, 358, 8-9), also die Möglichkeit, andere Länder zu erkunden und mit anderen Völkern Handel zu treiben. Sie kann daher nur in der zweiten Ebene - dem internationalen Recht - ihre Gewähr finden und geht ganz und gar in ihr auf.

\section{Die Gründe für eine Ablebnung}

Warum weigert sich Kant also, die domestic analogy zu besiegeln, das heißt, das Postulat des öffentlichen Rechts, das er für die Einzelmenschen geltend macht, auf die zwischenstaatlichen Beziehungen auszudehnen? Anders gefragt: Warum lehnt er den Völkerstaat in der Praxis ab, nachdem er in der Theorie seine Notwendigkeit anerkannt hat? Verstreut lassen sich in Kants Texten verschiedene Gründe dafür ermitteln. Bei einigen handelt es sich um Überlegungen empirischer Art: 1) Die übermäßige Ausdehnung eines Staates beeinträchtigt dessen Regierbarkeit (VI, 350, 1215); 2) eine »Universalmonarchie « würde die bürgerliche und politische Freiheit zu beschränken drohen (dass große Ausdehnung des Staates und Despotismus in Wechselbeziehung stünden, war eine gemeinsame Überzeugung der republikanischen Tradition des 17. und 18. Jahrhunderts, die auch Montesquieu teilte); 3) da der historische Fortschritt schließlich durch den »Antagonismus - die »ungesellige Geselligkeit « - angetrieben werde, müsse der internationale Frieden durch eine weltbürgerliche Ordnung gesichert werden, die, wie der Völkerbund, »nicht ohne alle Gefahr sei, damit die Kräfte der Menschen nicht einschlafen« (VIII, 26, 10-12). Aber solcherlei praktisch-empirische Betrachtungen haben in einer transzendentalen Rechtsauffassung, die das Rechtsgebäude ganz und gar a priori nach Maßgabe der allgemein gesetzgebenden reinen praktischen Vernunft errichtet, nicht den geringsten Wert.

Triftiger erscheint aufgrund seiner formalen Natur der Einwand, wonach die Staaten nicht gezwungen werden können, aus dem Naturzustand herauszufinden, in dem sie sich in ihren Wechselbeziehungen befinden, weil jeder von ihnen sich bereits in seinem Inneren eine Rechtsverfassung gegeben, den Naturzustand also hinter sich gelassen hat (VIII, 355, 36-37 - 356, 1). Allerdings haben auf diese Weise nur die Bürger ein und desselben Staates die Pflicht erfüllt, aus dem Naturzustand herauszutreten, nicht aber alle Individuen, die als Angehörige verschiedener Staaten miteinander in Kontakt kommen können und sich untereinander demnach weiterhin im Naturzustand befinden. Dagegen setzt das Postulat des öffentlichen Rechts »ein Recht [fest], jedermann, mit dem wir irgend auf eine Art in Verkehr kommen könnten, zu nöthigen, mit uns in eine Verfassung zusammen zu treten, worin jenes [ein äußeres Mein und Dein] gesichert werden kann« (VI, 256, 33-35). Im Übrigen widerspricht Kant sich selbst, wenn er in der Metaphysik der Sitten erklärt:

"Nun spricht die moralisch-praktische Vernunft in uns ibr unwiderstebliches Veto aus: Es soll kein Krieg sein, weder der, welcher zwischen Mir und Dir im Naturzustande, noch zwischen uns als Staaten, die, obzwar innerlich in gesetzli- 
chen, doch äußerlich (im Verbältniß gegen einander) im gesetzlosen Zustande sind $(V I, 354,20-25)$.

Die bisher betrachteten Begründungen mögen zwar sicher ernste Bedenken von Kant zum Ausdruck bringen, sie berühren aber nicht den Kern des Problems. Der wahre Grund für seine Ablehnung des Völkerstaats-Gedankens erhellt dagegen aus einer wichtigen Seite des Ewigen Friedens:

"Darin [im Begriff eines Völkerstaats, M. M.] aber wäre ein Widerspruch: weil ein jeder Staat das Verbältnis eines Oberen (Gesetzgebenden) zu einem Unteren (Geborchenden, nämlich dem Volk) enthält, viele Völker aber in einem Staate nur ein Volk ausmachen würden, welches [...] der Voraussetzung widerspricht" (VIII, 354, 8-15).

Kant führt das Argument so vor, als zeige es einen logischen Widerspruch auf, und zwar nach folgendem Gedankengang: Einerseits wohnt dem Begriff des internationalen Rechts die Idee der Pluralität der Völker als unbestreitbare Voraussetzung inne. Andererseits beinhaltet der universale Staat Einheit, Negation der Pluralität, das heißt Negation der Voraussetzung. Ist die Voraussetzung nach Kant aber notwendigerweise wahr, dann ist das ihr Widersprechende inakzeptabel.

In Wahrheit beinhaltet ein Völkerstaat indes keineswegs die Negation der Pluralität. Es gilt ihn nämlich streng vom homogenen Weltstaat, also von der Universalmonarchie abzugrenzen (wie Kant sich ausdrückt, der beide Begriffe wohl bisweilen verwechselt). In letzterer lösen sich die Staaten in einem einzigen universalen politischen Organismus auf, während der Völkerstaat nicht bloß ein »Staat von Individuen«, sondern von Völkern ist. Auch wenn folglich eine zentrale Bundesregierung existiert, die in einigen Bereichen (Außen-, Steuer-, Finanzpolitik) über Zwangsgewalt verfügt, bewahren die einzelnen Staaten dennoch ihre staatliche Identität und teilweise auch ihre Autonomie und Souveränität. Dies lehrte das Beispiel der Vereinigten Staaten, die von einem Staatenbund ohne Bundesregierung (Kongress in Philadelphia, 1774, der zu den 1777 verabschiedeten und 1781 in Kraft getretenen Articles of Federation führte) zu einem Bundesstaat mit Zentralregierung übergegangen waren (Verfassungskonvent von Philadelphia, 1787). Nun war Kant mit dem amerikanischen Fall gut vertraut, führte ihn in der Metaphysik der Sitten sogar als Negativbeispiel an (und zeigte in diesem Fall, dass er den Völkerstaat klar von der Universalmonarchie unterschied). Warum beharrt er also auf seiner Ablehnung des Völkerstaats und meint, er stehe im Widerspruch zur »Voraussetzung « der Staatenvielfalt? Tatsache ist, dass die »Voraussetzung «, von der Kant spricht, nicht theoretischer Natur ist, sondern aus einer empirischen Betrachtung des Bestehenden entspringt. Sie deckt sich eher mit der "ypothesis«, von der - wie wir gesehen haben - die zeitgenössischen Staaten ausgehen, die nicht bloß ihre politisch-administrative Identität, sondern ihre Unabhängigkeit und absolute Souveränität bewahren wollen, im Gegensatz zur »thesis « der Vernunft, die für die Gründung eines Völkerstaats sprechen würde. Mit ihnen teilt Kant die »Voraussetzung«-»ypothesis«, dass die Souveränität absolut unteilbar sei: entweder man besitzt sie ganz oder es ist keine Souveränität. 
Dass Kant dieser naturrechtsphilosophischen Konzeption, die das Prinzip der absoluten staatlichen Souveränität verteidigte, stark verhaftet blieb, obwohl er in anderer Hinsicht den Vertretern der Naturrechtsschule gegenüber kritisch eingestellt war, beweist auch sein persönliches Exemplar des Jus naturae in usum anditoru. Pars posterior von Gottfried Achenwall, der Text, den er als Handbuch für seine Vorlesungen benutzte. In Buch IV lesen wir: »Quaelibet gens respectu alterius gentis est persona moralis degens in statu naturali $(\$ 210)$. Daraus folgt: "gentes omnes sunt liberae et a se invicem independentes $(\$ 214)^{6}$. Achenwall geht davon aus, dass die Völker, oder einige von ihnen, sich in einem dauerhaften internationalen Bündnis (societas libera maior et aeterna, $\$ 190$ ) frei zusammenschließen, das entweder Zwangscharakter haben oder sich - was die eingehender analysierte Hypothese ist - auf eine Föderation (foederatarun rerum publicarum corpus, $\mathbb{} 211$ ) beschränken kann ${ }^{7}$. Derlei Bündnisse fallen seiner Ansicht nach aber nicht unter das Naturrecht, das jeder Nation absolute Unabhängigkeit sowie das Recht verleiht, für ihre Selbsterhaltung zu sorgen, vorausgesetzt das Naturrecht wird dadurch nicht verletzt. Bedeutsamerweise hat Kant, der die von ihm verwendeten Texte gewöhnlich mit Randbemerkungen vollschrieb, wenn er nicht einverstanden war, an diesem Teil von Achenwalls Handbuch nur wenige und eher unbedeutende Bemerkungen angebracht.

\section{Ein Kompensationsversuch}

Kant ist sich völlig darüber im Klaren, dass die Option für den Völkerbund und gegen den Völkerstaat eine Schwächung der Friedensgarantie bedeutet. Wahrscheinlich unternahm er deshalb seit 1793 einen Kompensationsversuch, indem er den Mechanismus der Friedenssicherung weniger dem internationalen Organismus als vielmehr der Umformung der inneren Verfassung der Einzelstaaten übertrug. Kants politisches Ideal war die republikanische Regierungsform, in der die Macht ausschließlich nach dem Gesetz, und damit (wenigstens ideell) gemäß dem Volkswillen ausgeübt werde. Gerade weil die republikanische Verfassung auf dem Volkswillen und nicht auf der Willkür des Despoten gründet, ist sie in seinen Augen tendenziell auf Frieden ausgerichtet.

»Wenn [...] die Bestimmung der Staatsbürger dazu erfordert wird, um zu beschließen, ob Krieg sein sollte, oder nicht, so ist nichts natürlicher, als daß, da sie alle Drangsale des Krieges über sich selbst beschließen müßten [...], sie sich sebr bedenken werden, ein so schlimmes Spiel anzufangen" (VIII, 351, 4-11).

6 G. Achenwall, Juris naturalis pars posterior, complectens Ius familiae, Ius publicum et Ius gentium in usum auditorum, in Kantss gesammelte Schriften (Akademie-Ausgabe), XIX, 419 e 421.

7 Vgl. Akademie-Ausgabe, XIX, 411 und 420. 
Die Friedenssicherung durch einen internationalen Organismus und das Eintreten für die republikanische Regierung sind zwei eng miteinander verknüpfte Pole von Kants rechtspolitischem Denken: Ohne republikanischen Geist (heute würden wir sagen: ohne Demokratie) kann der Friede nicht gefördert werden, wie umgekehrt der Republikanismus nicht ohne Frieden möglich ist. Nicht von ungefähr verkehrt sich jedoch das Ursache-Folge-Verhältnis zwischen beiden Faktoren im Verlauf der Kantischen Denkentwicklung. In der Idee von 1784, als er dem bindenden Handeln des internationalen Friedensbundes noch große Bedeutung beimaß - auch wenn er ihn nicht mit einer Zentralregierung gleichsetzte -, betrachtete Kant den Völkerbund als Voraussetzung für die Errichtung der republikanischen Verfassung:

"Das Problem der Errichtung einer vollkommnen bürgerlichen Verfassung ist von dem Problem eines gesetzmäßigen äußeren,Staatenverhältnisses abhängig und kann obne das letztere nicht ausgelöset werden" (VIII, 24, 2-5).

In der Schrift von 1795 kehrt Kant das Verhältnis dagegen um, nachdem er nunmehr seine Kritik am Völkerstaat verschärft hat. Der erste Definitivartikel (und folglich die erste Voraussetzung) für den ewigen Frieden lautet: »Die bürgerliche Verfassung in jedem Staate muß republikanisch sein « (VIII, 349, 8). Erst der zweite, vom ersten abhängende Artikel fordert: »das Völkerrecht soll auf einen Föderalismus freier Staaten gegründet sein« (VIII, 354, 2).

Mit Sicherheit war diese gewandelte Orientierung durch den Gang der Französischen Revolution mitbeeinflusst worden, auf die Kant - als einer der wenigen Deutschen seiner Zeit - auch nach Königsmord und Terror weiterhin seine Hoffnungen setzte.

"Die Ausführbarkeit (Objektive Realität) dieser Idee der Föderalität, die sich allmäblig über allen Staaten erstrecken soll und zum ewigen Frieden hinführt, läßt sich darstellen. Denn wenn das Glück so fugt: daß ein mächtiges und aufgeklärtes Volk sich zu einer Republik (die ibrer Natur nach zum ewigen Frieden geneigt sein muß) bilden kann, so giebt diese einen Mittelpunkt der föderativen Vereinigung für andere Staaten ab, um sich an sie anzuschließen und so den Freiheitszustand der Staaten gemäß der Idee des Völkerrechts zu sichern und sich durch mehrere Verbindungen dieser Art nach und nach immer weiter auszubreiten" (VIII, 356, 14-23).

Näher liegend ist aber, dass Kant die Gewähr für den Frieden, die er von einer auf der Voraussetzung der absoluten Souveränität der Staaten fußenden internationalen Ordnung nicht erwarten konnte, woanders - nämlich in der inneren Transformation der Staaten statt in ihrer äußeren Assoziation - zu suchen begann.

\section{Realismus oder Utopie?}

Kant ist ein revolutionärer Denker, was Erkenntnistheorie und Moral, was das politische Denken, aber auch was die Friedenslehre betrifft. Die Friedenspläne vor 
ihm machten gewöhnlich an den europäischen Grenzen Halt, waren in Wirklichkeit also bloße Erweiterungen des Hobbes«schen Modells, wobei man die pax civilis auf ganz Europa ausdehnte und den Naturzustand außerhalb seiner Grenzen verwies. Mit Hilfe seiner universalistischen Vernunftauffassung gelingt es Kant dagegen, einen die ganze Welt umschließenden Frieden zu denken. Was die Souveränitätsauffassung angeht, die er nicht anders als absolut und unteilbar zu denken vermag, bleibt er indes ein Kind seiner Zeit. Was den meisten heutzutage offensichtlich erscheint, dass die Staaten sich nämlich einem Integrationsprozess, der unweigerlich die fortschreitende Einbuße von »Stücken « von Autonomie und Souveränität mit sich bringt, nicht entziehen können, war für den »Naturrechtsphilosophen « Kant undenkbar - auch um den Preis, mit sich selbst, mit seiner Auffassung von Recht und reiner praktischer Vernunft in Widerspruch zu geraten.

Es ist sicher nicht leicht, Lösungen für das Problem eines Weltföderalismus aufzuzeigen, ohne in Utopie zu verfallen. Deshalb hat die Kritik sich häufig sehr nachgiebig gezeigt mit Kants Position, der gerade das Verdienst des Realismus zuerkannt wurde. Viele Interpreten betrachten Kants Option für den Völkerbund als eine Art vernunftbedingtes Sich-Abfinden mit einem leichter erreichbaren Ziel, während die ehrgeizigere Verwirklichung des Völkerstaats einer künftigen theoretischen Perspektive vorbehalten blieb. Diese Position birgt jedoch ein doppeltes Missverständnis. Erstens weil Kant an keiner Stelle schreibt, dass der Völkerbund vorerst die bessere Lösung sei. Im Gegenteil. Der Wortlaut der Texte zeigt unmissverständlich, dass er den Völkerbund (offenkundig im Sinn einer »Konföderation «) trotz der theoretischen Überlegenheit des Völkerstaats für die endgültige Lösung hält, weil sie als einzige der »Voraussetzung « der Pluralität autonomer und unabhängiger Staaten nicht widerspricht.

Aber es liegt ein zweites, noch weiter reichendes Missverständnis vor, das unmittelbar Kants Realismus betrifft. Es stimmt ganz genau, dass Kant kein Utopist war, wenn wir Utopie in der - in Wahrheit ein wenig reduktiven - Bedeutung der Verwechslung von projekthafter Idealität und tatsächlicher Realisierbarkeit verstehen. In diesem Sinne war beispielsweise das Projet pour rendre la paix perpétuelle en Europe (1713) des Abbé de Saint-Pierre utopisch, der glaubte, die Vernünftigkeit seines Projekts sei eine sichere Gewähr für dessen Realisierung, es reiche also, an die Vernunft zu appellieren, damit ausgerechnet die absoluten, keiner Kontrolle unterworfenen Gewalthaber fürderhin zugunsten des normativen Prinzips auf Gewaltanwendung verzichten würden. Kant war in diesem Sinne überhaupt kein Utopist, weil er den Abstand zwischen der faktischen Wirklichkeit und der Idealität seines Projekts von Grund auf zu würdigen wusste. In der Metaphysik der Sitten erklärt er auf der selben Seite, wo er den Bund zur Erhaltung des Friedens auf einen permanenten Staatenkongress reduziert, unumwunden, dass der ewige Friede "freilich eine unausführbare Idee« (VI, 350, 17) sei. Doch das Bewusstsein vom Abstand zwischen Realität und Idealität, von der unüberbrückbaren Kluft zwischen Sein und Sein-Sollen, kann im transzendentalen System der Absolutheit der Norm keinen Abbruch tun, die allen praktisch-faktischen Erwägungen zum Trotz absolut bleibt. 
"Also ist nicht mehr die Frage: ob der ewige Friede ein Ding oder Unding sei, und ob wir uns nicht in unserem theorethischen Urtheile betrïgen, wenn wir das erstere annehmen, sondern wir müssen so handeln, als ob das Ding sei, was vielleicht nicht ist... $(V I, 354,25-29)$.

Kants »Realismus « besteht also nicht in der Akzeptanz von Kompromisslösungen, sondern in seiner Einsicht, dass die Absolutheit der Norm und ihre mögliche Unrealisierbarkeit (bzw. nur asymptotische Realisierbarkeit) durchaus vereinbar sind. Obwohl oder gerade weil das Imperativische der Norm von ihrer Realisierbarkeit unabhängig ist, kann es nämlich zum Leitkriterium für die Rationalisierung der Wirklichkeit werden. Die Stärke von Kants rechtspolitischem Denken liegt im absoluten Wert des Normativen, das gerade dadurch konkret wirksam wird (also nicht utopisch ist), dass es die Kluft zwischen Realität und Idealität als unüberbrückbar anerkennt. Wie Kant selber uns gelehrt hat, brauchen wir für Überlegungen pragmatischer Art keine Philosophen, oder keine »moralischen Politiker «, welche die Politik der Moral unterordnen, sondern es reichen die »politischen Moralisten «, die die Theorie dem praktischen Nutzen anpassen.

\section{Zusammenfassung}

Kant dehnt das Vertragsmodell von der Ebene der Individuen auf die der Staaten aus. Nach der Meinung des Autors hätte Kant das Prinzip der heutzutage so genannten domestic analogy aus systematischen Gründen rigoros anwenden sollen: Zur Analogie mit der bürgerlichen Gesellschaft sollte der internationale Friedensorganismus ein mit zentraler Regierung und zentraler Gewalt ausgestatteter »Völkerstaat« sein. Aber Kant zieht ab 1795 immer entschiedener die Lösung des »Völkerbundes" vor, die die Autonomie und Unabhängigkeit der einzelnen Staaten garantiert. Die These des Aufsatzes ist folgende: Hinter dem von Kant festgestellten logischen Widerspruch zwischen dem Völkerstaat und dem Begriff des Völkerrechts steckt Kants Festhalten an der naturrechtlichen Tradition und am zeitgenossischen dogmatischen Prinzip der absoluten Staatssouveränität. Ab der Mitte der Neunziger Jahre fängt Kant deshalb an, lieber den Frieden vom inneren Republikanisierungsprozess der Staaten zu erwarten als die Errichtung der republikanischen Verfassung von internationalen Friedensinstitutionen.

\section{Summary}

Kant extends the model of social contract from the individual to the international level. The author's opinion is that, to be consistent with his transcendental philosophy of law, Kant should have strictly applied the principle of »domestic analogy «, as today political scientists say. The international organization for peace should be a real »State of peoples « with a central government and a central power, in analogy to the civil society. However, since 1795, Kant prefers the solution of the »Federation of peoples «, which warrants the autonomy and the independence of the single Sta- 
tes. The article's point is the following: Kant founds his refusal of the State of peoples on its apparent logical contradiction with the concept of Law of nations, which would foreground a plurality of peoples; actually he cannot free himself from the tradition of Natural Law and from the dogmatic principle of absolute sovereignty of States. Since the mid Nineties he begins to expect peace from the inward transformations of States towards republican constitution instead of considering international institutions for peace as committing republicanism.

\title{
Friedensnobelpreis 2005
}

\section{für internationalen Einsatz gegen Verbreitung von Atomwaffen und für größere nukleare Sicherheit}

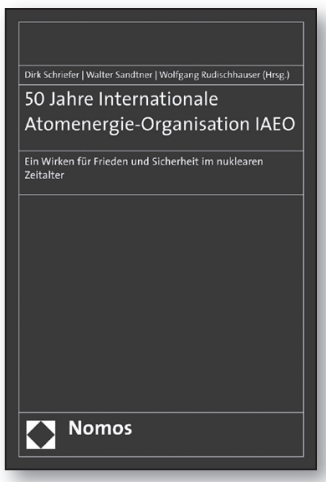

\author{
50 Jahre Internationale Atomenergie- \\ Organisation IAEO \\ Ein Wirken für Frieden und Sicherheit \\ im nuklearen Zeitalter \\ Herausgegeben von Dr. Dirk Schriefer, \\ MinR Dr. Walter Sandtner, Bundesministerium \\ für Wirtschaft und Technologie und Dipl.-Kfm. \\ Wolfgang Rudischhauser, Vortragender \\ Legationsrat, Leiter der Arbeitseinheit IAEO \\ im Auswärtigen Amt \\ 2006, 218 S., brosch., 29,-€, ISBN 3-8329-2465-5
}

Friedensnobelpreis 2005 für die IAEO und ihren Generaldirektor - für die Sicherung der Nuklearenergie und ihrer Anwendungen vor militärischem Missbrauch und zur Verbesserung der Sicherheit bei ihrer friedlichen Nutzung. Zum Repertoire der IAEO gehören neben den Schlaglichtern Iran, Irak und Nordkorea auch der Umweltschutz, Ernährung, die medizinische Versorgung und sauberes Trinkwasser.

Bitte bestellen Sie bei Ihrer Buchhandlung oder bei: 